${ }^{1}$ H. Metzner, B. Metzner, and M. Calvin, Arch. Biochem. and Biophys. (in press).

${ }^{2}$ H. Metzner and B. Metzner, University of California Radiation Laboratory Report, UCRL 3710 (March, 1957), p. 4; H. Metzner, H. Simon, B. Metzner, and M. Calvin, these Proceedings, 43, $892,1957$.

${ }^{3}$ H. Metzner, H. Simon, and B. Metzner, University of California Radiation Laboratory Report, UCRL 3950 (September, 1957), p. 5.

4 H. Metzner, Planta, 45, 493, 1955.

5 C. Van Baalen, H. S. Forrest, and J. Myers, these Proceedings, 43, 701, 1957.

${ }^{6}$ B. K. Bachhawat, J. F. Woessner, Jr., and M. J. Coon, Federation Proc., 15, 214, 1956.

${ }^{7}$ C. R. Greenberg, Federation Proc., 13, 221, 1954.

8 J. R. Quayle, R. C. Fuller, A. A. Benson, and M. Calvin, J. Am. Chem. Soc., 76, 3610, 1954; M. Calvin and P. Massini, Experientia, 8, 445, 1952; A. Weissbach, B. L. Horecker, and J. Hurwitz, J. Biol. Chem., 218, 795, 1956; E. Racker, Nature, 175, 249, 1955.

${ }^{9}$ E. Racker, "The Reductive Pentose Phosphate Cycle. I. Phosphoribulokinase and Ribulose Diphosphate Carboxylase," J. Biol. Chem. (in press); N. G. Pon, thesis, University of California, Berkeley, 1958.

10 M. Flavin, H. Castro-Mendoza, and S. Ochoa, Biochim. et Biophys. Acta, 20, 591, 1956.

${ }^{11}$ O. Warburg and J. Kripphal, Z. Naturiorsch., 11b, 718, 1956.

\title{
THE NATURE OF BOND ORBITALS AND THE ORIGIN OF POTENTIAL BARRIERS TO INTERNAL ROTATION IN MOLECULES
}

\section{By Linus Pauling}

GATES AND CRELLIN LABORATORIES OF CHEMISTRY, CALIFORNIA INSTITUTE OF TECHNOLOGY,
PASADENA*

Communicated December 16, 1957

Twenty years ago Kemp and Pitzer ${ }^{1}$ discovered that the relative rotation of the two methyl groups about the carbon-carbon bond in ethane is not completely free but is restricted by a potential barrier about $3.0 \mathrm{kcal} / \mathrm{mole} \mathrm{high,} \mathrm{with} \mathrm{three} \mathrm{maxima}$ and three minima in a complete rotation, corresponding to the trigonal symmetry of the methyl groups. It was soon found that rotation about single bonds is restricted in many molecules, and many experimental values for the height of the potential barrier have been obtained by the analysis of thermodynamic quantities (entropy, heat capacity) and especially by the methods of microwave spectroscopy.

Progress has been slow in the development of a satisfactory theory for the potential barriers, despite the efforts of several investigators. The theoretical attack was begun by Eyring, ${ }^{2}$ who made approximate quantum-mechanical calculations of the interactions of the hydrogen atoms of the two methyl groups. The various suggestions and calculations about the importance of van der Waals repulsion between attached groups, electrostatic interactions of the charge distributions in the bonds between the two carbon atoms and the attached groups, and intrinsic lack of cylindrical symmetry in the axial chemical bond itself have been summarized recently by Wilson, ${ }^{3}$ who tested these hypotheses by comparison with the values of the potential barriers that have been determined experimentally and reached the conclusion that the potential barriers to internal rotation "must in some way be an inherent property of the axial bond itself and not due in any substantial measure to direct forces between the attached atoms or those parts of the electron distribution which are out on the attached bond any considerable distance." References to the 
earlier theoretical papers and to the sources of most of the values of potential barriers mentioned later in this paper are given by Wilson.

I have developed a simple theory of these potential barriers, described in the following paragraphs. According to this theory, the potential barriers are not a property of the axial bond itself, but result from the exchange interactions of electrons involved in the other bonds (adjacent bonds) formed by each of the two atoms, as determined by the overlap between the parts of the adjacent bond orbitals that extend from each of the two atoms toward the other.

Concentration of Bond Orbitals. - The four bonds formed by a carbon atom can be discussed with use of tetrahedral orbitals formed by hybridization of the $s$ orbital and the three $p$ orbitals of the neon shell. ${ }^{4,5}$ The description of these orbitals as $s p^{3}$ tetrahedral orbitals is, however, only a first approximation. Even for the hydrogen atom, for which the $1 s$ orbital is far more stable than any other orbital, hybridization occurs on bond formation. This effect has been described ${ }^{5}$ as concentration of the bond orbitals. For example, in the approximate variation treatment of the hydrogen molecule by Wang ${ }^{6}$ and the corresponding treatment of the hydrogen moleculeion by Finkelstein and Horowitz, ${ }^{7}$ use is made of an orbital that has the form of a $1 \mathrm{~s}$ orbital but with changed effective nuclear charge, found by minimization of the energy to be 1.17 and 1.23 , respectively. The change in the value of the effective nuclear charge is equivalent to hybridizing the $1 s$ orbital of the hydrogen atom with the $2 s, 3 s$, and higher orbitals, including $s$ orbitals in the continuum. These higher $s$ orbitals make a total contribution of 2 per cent to the hybrid bond orbitals. Also, a significant improvement in the approximate wave function can be obtained by adding to the $s$ orbital a term containing a cosine function, which we may describe as a $p$ orbital. This $p$ term is a hybrid of $2 p, 3 p$, and higher $p$ orbitals. The effective nuclear charge that minimizes the energy is about twice that for the $s$ orbital, leading to approximately the same spatial extent. It was shown by Rosen ${ }^{8}$ for the hydrogen molecule and by Dickinson ${ }^{9}$ for the hydrogen molecule-ion that the energy is minimized when the $p$ terms occur in the wave function with coefficients 0.10 and 0.15 , respectively, corresponding to 1 and 2 per cent of $p$ character in the hybrid orbitals. These amounts of $p$ hybridization are found, despite the fact that the promotion energy for the $p$ terms is about $330 \mathrm{kcal} / \mathrm{mole}$, slightly larger than the ionization energy of the hydrogen atom, $313 \mathrm{kcal} / \mathrm{mole}$. The promotion energy for the higher $s$ terms is also about $330 \mathrm{kcal} / \mathrm{mole}$. (I am indebted to Dr. Brian Gray for carrying out the expansions of the wave functions in the set of hydrogen-atom functions with effective nuclear charge unity, and evaluating the promotion energies.)

We may accordingly expect that the bond orbitals of the carbon atom will be found on careful examination to be hybrids with some $d, f$, etc., character, in addition to the main $s p^{3}$ tetrahedral character. A rough estimate of the contributions of $d$ and $f$ terms to the hybrid bond orbital can be made by assuming the bond energy to be proportional to the strength of the bond orbital, as determined by the angular distribution, ${ }^{5}$ and taking the promotion energy for both $d$ and $f$ as equal to the ionization energy of the carbon atoms. This calculation leads to coefficients approximately 0.15 for both the $d$ term and the $f$ term, corresponding to about 2 per cent $d$ character and 2 per cent $f$ character in the tetrahedral orbitals.

The calculation is made in the following way. The bond orbital is taken to be 


$$
\Psi=\alpha s+\beta p_{z}+\gamma d_{z}+\delta f_{z},
$$

in which $s, p_{z}, d_{z}$, and $f_{z}$ are the orbitals with maximum values along the $z$-axis (the bond direction); normalized to $4 \pi$, their angular parts are

$$
\begin{aligned}
& s=1, \\
& p_{z}=\sqrt{3} \cos \theta \\
& d_{z}=\sqrt{5 / 4}\left(3 \cos ^{2} \theta-1\right), \\
& f_{z}=\sqrt{7 / 4}\left(5 \cos ^{3} \theta-3 \cos \theta\right)
\end{aligned}
$$

The value of this function in the bond direction, called the "strength of the bond orbital," is

$$
S=\alpha+\sqrt{3} \beta+\sqrt{5} \gamma+\sqrt{7} \bar{\delta}
$$

The effective bond energy is assumed to be the product of $S$ and a bond-energy parameter $b$, corrected by subtracting the terms $\gamma^{2} P_{d}$ and $\delta^{2} P_{f}$, in which $P_{d}$ and $P_{f}$ are the promotion energies for the $d$ and $f$ terms:

$$
\text { Bond energy }=b S-\gamma^{2} P_{d}-\delta^{2} P_{f}
$$

The value of $b$ is taken to be $36 \mathrm{kcal} / \mathrm{mole}$, which leads to bond energy $82 \mathrm{kcal} / \mathrm{mole}$, corresponding to an average bond formed by a carbon atom $(\mathrm{C}-\mathrm{C}, 82.3 ; \mathrm{C}-\mathrm{H}$, 99.6; C-O, 81.0; etc.). The promotion energies are taken to be $P_{d}=300 \mathrm{kcal} /$ mole and $P_{f}=350 \mathrm{kcal} / \mathrm{mole}$, somewhat larger than the ionization energy of carbon, $260 \mathrm{kcal} / \mathrm{mole}$, as indicated by consideration of the energies of the lowest $s, p$, $d, f$ Epstein functions for the hydrogen atom. ${ }^{10}$ In accordance with the minimumenergy theorem of quantum mechanics, we vary the parameters $\gamma$ and $\delta$ to make the bond energy a maximum. (A satisfactory approximation is to take $\alpha=1 / 4$; then $\beta^{2}=3 / 4-\gamma^{2}-\delta^{2}$.) It is found that both $\gamma$ and $\delta$ then have the value 0.15 , with $\beta=0.84$, and the bond orbital has the simple angular dependence

$$
\psi=0.33+0.85 \cos \theta+0.50 \cos ^{2} \theta+\cos ^{3} \theta
$$

This function has a maximum value (bond strength) of 2.68 , which is 34 per cent greater than for an $s p^{3}$ orbital-a large effect of 2.25 per cent $d$ character and 2.25 per cent $f$ character. The nodal cone lies at $113.8^{\circ}$ from the bond direction, only slightly shifted from the $s p^{3}$ value $109.5^{\circ}$. The negative lobe contains 7 per cent of the distribution function $\psi^{2}$, considerably less than the 11 per cent for $s p^{3}$ orbitals. The value of the function is larger than that of the $s p^{3}$ tetrahedral orbital from the bond axis out to $44^{\circ}$, and then becomes smaller, being about two-thirds as great (less than half the electron density) from $65^{\circ}$ to $140^{\circ}$. The concentration of the orbital in the bond direction is well described by the average value of $\cos \theta$ (weighted with the distribution function $\psi^{2}$ ), which is 0.771 for function 4 , and only 0.312 for an $s p^{3}$ orbital.

The hybrid orbital has cylindrical symmetry, and accordingly the introduction of $d$ character and $f$ character in the axial bond itself does not lead to an interaction dependent on the relative azimuthal orientation of the two groups.

The Interaction of the Concentrated Hybrid Orbitals. - The interaction of the two 
electrons occupying one adjacent bond orbital of each of two atoms forming an axial bond can be discussed by expanding the adjacent bond orbitals in terms of orbitals described with reference to the axial bond direction, which may be taken as the $z$-axis. The interaction energy of these two electrons (which with two other electrons, on adjacent atoms, form bonding electron pairs) includes the resonance integral ${ }^{11}$ with the coefficient $-1 / 2$ (rather than the +1 that occurs in the energy for a bond), and accordingly the negative sign of the resonance integral itself leads to repulsion between the two electrons and maximum stability when the overlapping is a minimum. In the expansion in functions about the bond direction there will occur $\sigma$ terms (independent of the azimuthal angle $\varphi$ ), $\pi$ terms (with factor $\cos$ $\varphi$ or $\sin \varphi), \delta$ terms $(\cos 2 \varphi)$, and $\phi$ terms $(\cos 3 \varphi)$. The interaction of two functions with $\sigma$ and $\phi$ character gives rise to an interaction energy proportional to $\cos 3 \varphi$, where $\varphi$ represents the relative azimuthal orientation of the two groups. This is the term of lowest order that occurs if one of the groups has trigonal symmetry (such as the methyl group).

A very rough estimate of the height of the potential barrier may be made. The $f$ term in an adjacent bond orbital as given in equations (1) and (4), which has its axis in a direction $70.5^{\circ}$ from the $z$-axis determined by the axial bond, can be expanded in terms of $f$ orbitals referred to this $z$-axis, and it is found that the coefficient of the normalized $f$ orbital $\sqrt{35 / 8} \sin ^{3} 6 \cos 3 \varphi$ is $0.664 \delta$, or 0.100 for $\delta=$ 0.150. The strength of this orbital is 2.09 , and hence it contributes 0.209 to the strength of the hybrid bond orbital. We may expect the corresponding term in the resonance integral to be approximately $(0.209 / 1.732)^{2}$ times as great as for two $\pi$ orbitals, as in the carbon-carbon double bond, which is about $61 \mathrm{kcal} / \mathrm{mole}^{12}$ Taking into account also the factor $-1 / 2$ for non-bonding rather than bonding interaction, we obtain $0.44 \mathrm{kcal} / \mathrm{mole}$. Each of the three $\mathrm{C}-\mathrm{H}$ bonds of one methyl group interacts with each of the three of the other group; hence we predict that the potential barrier for ethane and substituted ethanes (forming single bonds only) should be about $9 \times 0.44=4.0 \mathrm{kcal} / \mathrm{mole} \mathrm{high}$. This value is completely unreliable, and no significance should be attached to its rough agreement with the experimental values 2.7-3.0 kcal/mole for ethane, 3.30 for $\mathrm{CH}_{3} \mathrm{CH}_{2} \mathrm{~F}, 3.18$ for $\mathrm{CH}_{3} \mathrm{CHF}_{2}$, and 3.3 for propane.

The smaller value $2.56 \mathrm{kcal} / \mathrm{mole}$ found for $\mathrm{CH}_{3} \mathrm{CHOCH}_{2}$ shows the decrease that we would expect to result from the change in bond angles caused by the threemembered ring. If the change is distributed equally over the five other bond angles, increasing them from $109.5^{\circ}$ to $120^{\circ}$, the expansion of the $f$ orbital introduces the coefficient $0.514 \delta$ in place of $0.664 \delta$ and hence leads to a predicted barrier 77 per cent as great as for unstrained substituted ethanes, in good agreement with experiment $(2.56=0.77 \times 3.32)$.

There are other predictions that can be made on the basis of this theory, and there is further good agreement with experiment. First, it is predicted that the staggered configuration of substituted ethanes and similar molecules is the stable one. The staggered configuration has been verified for $\mathrm{CH}_{3} \mathrm{CH}_{2} \mathrm{Cl}, \mathrm{CH}_{3} \mathrm{CF}_{3}, \mathrm{CH}_{3} \mathrm{SiH}_{3}$, $\mathrm{CH}_{3} \mathrm{SiH}_{2} \mathrm{~F}, \mathrm{CH}_{3} \mathrm{SiF}_{3}$, and several other molecules.

Also, the height of the barrier changes from molecule to molecule in the predicted way. The barrier interaction involves the same integral over the radial parts of the wave functions as the axial bond itself, and it would accordingly be expected that 
for molecules in which the bond orbitals have similar hybrid character the barrier height would be a constant fraction of the bond energy. In particular, for different substituted ethanes essentially the same barrier would be found, provided that the substituent groups are not large enough to cause steric effects, which would increase the barrier height. Approximate constancy is observed for ethane and substituted ethanes, as mentioned above.

The energy of a carbon-silicon bond and that of a carbon-germanium bond are about three-quarters as great as that of a carbon-carbon bond, and it would accordingly be predicted that the height of the barrier in molecules containing these bonds would be about three-quarters as great, that is, about $2.3 \mathrm{kcal} / \mathrm{mole}$; observed values are 1.70 for $\mathrm{CH}_{3} \mathrm{SiH}_{3}, 1.56$ for $\mathrm{CH}_{3} \mathrm{SiH}_{2} \mathrm{~F}, 1.32$ for $\mathrm{CH}_{3} \mathrm{SiHF}_{2}$, and 2.5 for $\mathrm{CH}_{3} \mathrm{GeH}_{3}$ (perhaps uncertain). (Professor E. B. Wilson, Jr., has just written me that the value for methyl germane is probably 1.2.)

Only bond orbitals, and not orbitals for unshared pairs, hybridize with $f$ character, and accordingly we predict that an $\mathrm{OH}$ group would interact with a methyl group one-third as strongly as a methyl group itself would, and an $\mathrm{NH}_{2}$ group would interact two-thirds as strongly; for $\mathrm{CH}_{3} \mathrm{OH}$ and $\mathrm{CH}_{3} \mathrm{NH}_{2}$ we predict barrier heights about 1.0 and $2.0 \mathrm{kcal} / \mathrm{mole}$, respectively; the observed values are 1.07 and 1.90 .

We may describe the double bond as involving two tetrahedral orbitals of each of the two bonded atoms; that is, as two bent single bonds. ${ }^{5}$ It would then be expected that the interaction energy of a methyl group with a carbon atom forming a single bond and a double bond would be somewhat smaller than that between two methyl groups, that is, somewhat smaller than $3 \mathrm{kcal} / \mathrm{mole}$. The observed barrier height for methyl ethylene $\left(\mathrm{CH}_{3}-\mathrm{CH}=\mathrm{CH}_{2}\right)$ is $1.98 \mathrm{kcal} / \mathrm{mole}$, in agreement with expectation. Similar values are found in the related substances $\mathrm{CH}_{3}$ $\mathrm{CH}=\mathrm{C}=\mathrm{CH}_{2}$ (1.59) and $\mathrm{CH}_{3}-\mathrm{CH}=\mathrm{CHF}$ (2.15). Configurations have not been determined for these substances; we may predict that a hydrogen atom of the methyl group will be staggered with respect to the single bond on the other carbon atom.

In aldehydes and related substances the height of the potential hump is somewhat less than in methyl ethylene: $1.15 \mathrm{kcal} /$ mole for $\mathrm{CH}_{3} \mathrm{CHO}, 1.08$ for $\mathrm{CH}_{3} \mathrm{CFO}, 1.35$ for $\mathrm{CH}_{3} \mathrm{CClO}$, and 1.27 for $\mathrm{CH}_{3} \mathrm{COCN}$. Configurations in agreement with our prediction have been reported for the first three, a methyl hydrogen atom being staggered with respect to the single bond formed by the other carbon atom.

For acetic acid, $\mathrm{CH}_{3} \mathrm{COOH}$, in which the bond from carbon to the $\mathrm{OH}$ group has about 35 per cent double-bond character and that to the oxygen atom about 65 per cent double-bond character, as determined from the interatomic distances, we would predict for the potential hump a value about one-third that in acetaldehyde. This prediction is borne out by experiment, the height of the barrier being 0.48 $\mathrm{kcal} / \mathrm{mole}$.

For nitromethane, $\mathrm{CH}_{3} \mathrm{NO}_{2}$, and methyl difluoroborane, $\mathrm{CH}_{3} \mathrm{BF}_{2}$, symmetry requires that the potential barrier correspond to a sixfold axis. The experimental values for the barrier height are very small, 0.006 and $0.014 \mathrm{kcal} / \mathrm{mole}$, respectively. These very small values show that the contributions of the corresponding orbitals to the hybrid bond orbitals are also very small. The orbitals that could give rise to a potential barrier proportional to $\cos 6 \varphi$ are the $i$ orbitals, with azimuthal quantum number $l=6$. Because of the many nodal cones and the large promotion energy 
of the $i$ orbitals, it is to be expected that they would not make any important contribution to the hybrid bond orbitals.

The general qualitative agreement with experiment provides support for the theory that the potential barriers to internal rotation result from the interaction of adjacent hybrid bond orbitals with a small amount of $f$ character. The magnitude of the potential barriers, about 4 per cent of the energy of the axial bond in case that there are three interacting bonds on each of the two atoms and proportionately less for a smaller number of bonds, is also reasonable. A detailed quantummechanical treatment of restricted rotation carried out along the lines sketched here should yield results that would permit a detailed test of the theory to be made; in the meantime I believe that the above simple treatment and the extensive empirical support of the theory provide justification for it.

* Communication No. 2285.

${ }^{1}$ J. D. Kemp and K. S. Pitzer, J. Am. Chem. Soc., 59, 276, 1937.

${ }^{2}$ H. Eyring, J. Am. Chem. Soc., 54, 3191, 1932.

${ }^{3}$ E. B. Wilson, Jr., these Proceedings, 43, 816, 1957.

${ }^{4} \mathrm{~L}$. Pauling, these Proceedings, 14, 359, 1928.

5 L. Pauling, J. Am. Chem. Soc., 53, 1367, 1931.

${ }^{6}$ S. C. Wang, Phys. Rev., 31, 379, 1928.

${ }^{7}$ B. N. Finkelstein and G. E. Horowitz, Z. Phys., 48, 118, 1928.

${ }^{8}$ N. Rosen, Phys. Rev., 38, 2099, 1931.

9 B. N. Dickinson, J. Chem. Phys., 1, 317, 1933.

${ }^{10}$ P. S. Epstein, Phys. Rev., 28, 695, 1926.

11 J. C. Slater, Phys. Rev., 38, 1109, 1931.

12 B. S. Rabinovitch, J. E. Douglas, and F. S. Looney, J. Chem. Phys., 20, 1807, 1952; $23,315$. 1955.

\section{IDENTIFICATION OF BLOOD CHARACTERISTICS COMMON TO ALCOHOLIC MALES*}

By Roger J. Williams, Richard B. Pelton, Hertta-Maija Hakkinen, and LORENE L. ROGERS

CLAYTON FOUNDATION FOR RESEARCH, BIOCHEMICAL INSTITUTE, AND DEPARTMENT OF CHEMISTRY, UNIVERSITY OF TEXAS, AUSTIN, TEXAS

Communicated December 9,1957

In 1947 our laboratory began the intensive study of alcoholism, on the presumption that individual differences in metabolism are basic to the etiology of the disease and that "no psychological stresses can make an individual an alcoholic unless he has inherited a metabolic pattern which renders him susceptible." ${ }^{1}$ This working hypothesis-so designated at the time-has held our constant interest.

The difficulties of testing this hypothesis have been great, mainly because at the start almost complete ignorance existed as to how one individual might differ from another metabolically. Very few investigators have had any specific interest in gathering this type of information. Recently the senior author has attempted to assemble in a book entitled Biochemical Individuality ${ }^{2}$ the available material on the 